Mr. John Sauter

Dr. Dylan P. Brennan

Department of Physics

University of Tulsa

800 South Tucker Drive

Tulsa, OK 74104

Dr. Curt Bolton

Dr. John Mandrekas

U.S. Department of Energy

Office of Fusion Energy Sciences

19901 Germantown Road

Germantown, MD 20874-1290

April 24, 2013

Re: Final report on grant DE-FG02-08ER54950 titled "Flow Shear Effects in the Onset Physics of Resistive MHD Instabilities in Tokamaks"

Dear Mr. Sauter, Dr. Bolton, and Dr. Mandrekas,

After funding this project for two years, plus two additional years of no-cost extension, this project contributed significantly to establishing a foundation for continuing research in my team. This research project focused on one main thrust: understanding flow shear effects between resonant instabilities in tokamaks. This grant funded summer months and teaching time for myself as PI, one graduate student, travel and other support to address this research. The grant was extended at no cost after two years of the initial period 2/1/08-1/31/11 were funded, at which time a new grant DE-SC0004125 was started in 2010, which superseded this initial project. The research progress that was made in this initial project has been significantly impactful on that subsequent research as well as the work of several of my colleagues, and continues to contribute to our work today. Below I have summed up the research progress that was made for your review.

Timeline and Evaluation Phases

Year 1: Develop an analysis of resistive mode onset in selected DIII-D and NSTX experimental cases, including accurate equilibrium reconstructions and linear analyses. Begin linear analyses of flow inclusive equilibria with NIMROD in comparison to results from PEST-III.

Year 2: Extend the previous analyses to focus on aspect ratio effects using model equilibria in comparison with experimental cases. Study the nonlinear coupling between modes in nonlinear simulations.

The third year was not funded under this grant, but for completeness it is listed. Clearly, from the progress overview below, to a large extent it was completed:

Year 3: Study linear onset and nonlinear evolution of resistive modes in tokamaks, in comparison to experimental cases, including flow shear effects, using reduced analytic models, linear codes, and NIMROD. 


\section{Progress Overview}

The progress in this research centers around a focal point of a draft publication on the computational analysis of flow shear effects in the onset of a 3/2 mode driven by a $1 / 1$ mode in DIII-D equilibria. The paper itself remains in draft, but the knowledge gained from the effort, and the results, contributed to several other published papers. The initial idea was to try and calculate, via nonlinear simulations with NIMROD, the effects of rotation shear on driven $3 / 2$ and $2 / 1$ seed island physics, in experimentally relevant DIIID equilibria. The study is set up as an extension of work originally published in 2003 and 2005.

D.P. Brennan, S.E. Kruger, T.A. Gianakon, and D.D. Schnack, "A Categorization of Tearing Mode Onset in Tokamaks via Nonlinear Simulation," Nucl. Fusion 451178 (2005).

D.P. Brennan, R.J. La Haye, A.D. Turnbull, M.S. Chu, T.H. Jensen, L.L. Lao, T.C. Luce, P.A.

Politzer, E.J. Strait, S.E. Kruger, and D.D. Schnack, "A Mechanism for Tearing Onset Near Ideal Stability Boundaries," Phys. Plasmas 10, 1643 (2003).

In which a DIII-D discharge (086166) was studied in a stability analysis that explained why the $3 / 2$ neoclassical tearing mode was driven unstable by a sawtooth crash. The linear stability of the $3 / 2$ mode was becoming less stable and the sawtooth crashes, which remained of the same size and drove similar $3 / 2$ seed island sizes, eventually set off a $3 / 2$ NTM. In this work one important question was the effect of flow shear on this mechanism. In addition to direct simulations, several analytic efforts complemented this work and simple predictions suggested significant seed islands were expected in nonlinear simulations that more closely approached experimental conditions.

In the research funded by this grant, however, the simulations indicated that very small seed islands were directly driven, as shielding between the sawtooth and the surfaces is significant at the high Lundquist numbers of the experiment. Instead, long after the initial crash the difference in linear stability of the $3 / 2$, which remained prevalent despite the flattening of the core profiles from the sawtooth, contributed to a difference in the eventual seed island evolution. Essentially the seed islands grew or decayed long after the sawtooth crash, and not directly from it. The small size of the driven current can be seen in the attached figures.

These results were first reported in a private communication to Dr. Scott Kruger (TechX) on Oct. 27, 2009:

D.P. Brennan, S.E. Kruger and R.J. La Haye "Toroidal flow effects in nonlinearly driven resistive plasma instabilities"

The reason this paper on the simulated sawtooth crash was not published as planned was mainly one of time available to refine the computational work to fully support the physics arguments. In the past few years, computational developments have made these simulations more accessible, making it more likely that this paper will be completed under the subsequent funding of DE-SC0004125.

Several conference presentations (see above and for more detail http://www.personal.utulsa.edu/ dylan-brennan) were devoted to this effort to bring the community's opinions to bear on the details involved. Effectively the dominant 1/1 mode was found to be dragging the coupled modes surrounding it at a high rate through the 
plasma at their surfaces. The $1 / 1$ mode is locked to the local frame of the plasma in the core, where the flow rate is greatest. The resonant perturbations at the surrounding surfaces propagate in the "high slip regime" in the language of Fitzpatrick. Peaked flux averaged jxb forces (see Figs. 1 and 2) agree with localized flow modifications at the surfaces in analogy with Ebrahimi, PRL 2007. We track the mode into nonlinear saturation and have found oscillatory states in the evolution. During a visit (11/09) to Tulsa by R.J. LaHaye (GA), it became clear that similar oscillatory states are observed in DIII-D for these types of discharges. Including such a comparison would make this a very high impact study.

The understanding of the physics of the shielding and flow shear effects that we gained in these studies contributed at least in part to the following subsequent papers:

R.J. La Haye, R.J. Buttery, S.P. Gerhardt, S.A. Sabbagh, and D.P. Brennan "Aspect ratio effects on neoclassical tearing modes from comparison between DIII-D and NSTX," Phys. Plasmas 19, 062506 (2012).

R.J. La Haye, D.P. Brennan, R.J. Buttery, and S.P. Gerhardt, "Islands in the Stream: The Effect of Plasma Flow on Tearing Stability," Phys. Plasmas 17, 056110 (2010).

S.P. Gerhardt, D.P. Brennan, R. Buttery, R.J. La Haye, S. Sabbagh, E. Strait, M. Bell, R. Bell, E. Fredrickson, D. Gates, B. LeBlanc, J. Menard, D. Stutman, K. Tritz, and H. Yuh, "Relationship Between Onset Thresholds, Trigger Types and Rotation Shear for the $\mathrm{m} / \mathrm{n}=$ 2/1 Neoclassical Tearing Mode in a High- $\beta$ Spherical Torus," Nucl. Fusion 49, 032003 (2009).

R.J. La Haye, P.A. Politzer, and D.P. Brennan, "Beta Limit Due to $\mathrm{m} / \mathrm{n}=2 / 1$ Tearing Mode Onset in the DIII-D Hybrid Scenario," General Atomics Report 025707, Nucl. Fusion 48, 015005 (2008).

In these papers the work was not directly due to the nonlinear simulations, but instead had linear stability analyses and contributions in understanding the Rutherford modeling gained from the nonlinear simulation effort.

A large fraction of the time awarded at NERSC on m908 was used for the research on this grant.

Graduate Students

- Two students were at least partly supported by this grant. Hadi Mohammadi completed a masters thesis on neoclassical transport effects on Rutherford island evolution, ala S. Guenter. These results could lead directly lead to publication. Michael Halfmoon is currently working with energetic particle effects on resistive MHD modes, including work with PEST-III and NIMROD, and is now funded on a new grant. 


\section{Figures}
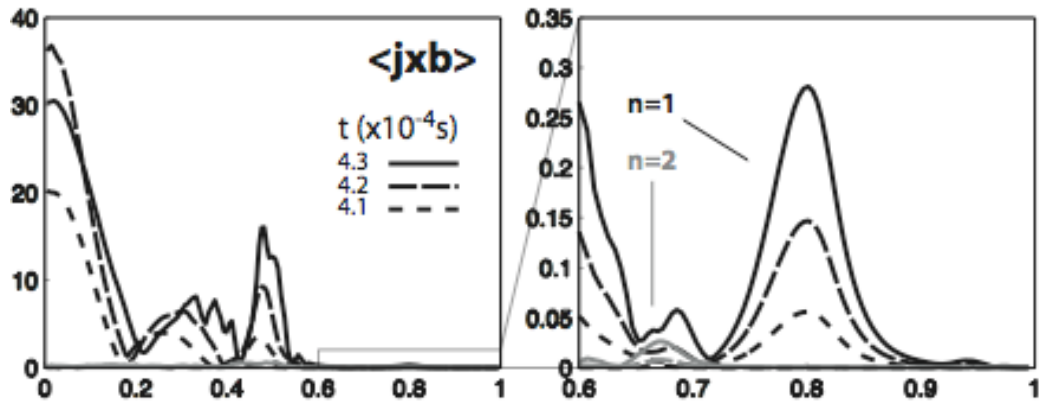

Fig. 1. The absolute value of the flux averged $\langle j x b\rangle$, vs. $\rho$ for the $n=1$ and $n=2$ modes on entry to the saturated stage in a DIII-D simulation with a $\mathrm{q}=1$ mode surrounded by resonant surfaces with flow shear.
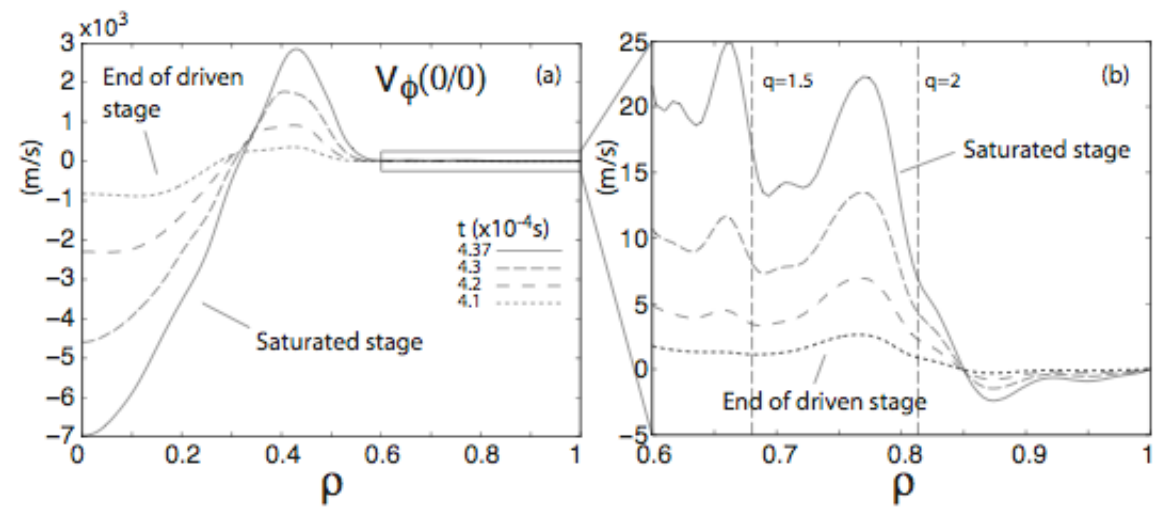

Fig. 2 . The $m / n=0 / 0$ flow perturbation on entry to the saturated stage. Despite significant flow modifications, the $2 / 1$ and $3 / 2$ saturated modes propagate rapidly in the plasma frame, while the $1 / 1$ is locked in the plasma frame. 

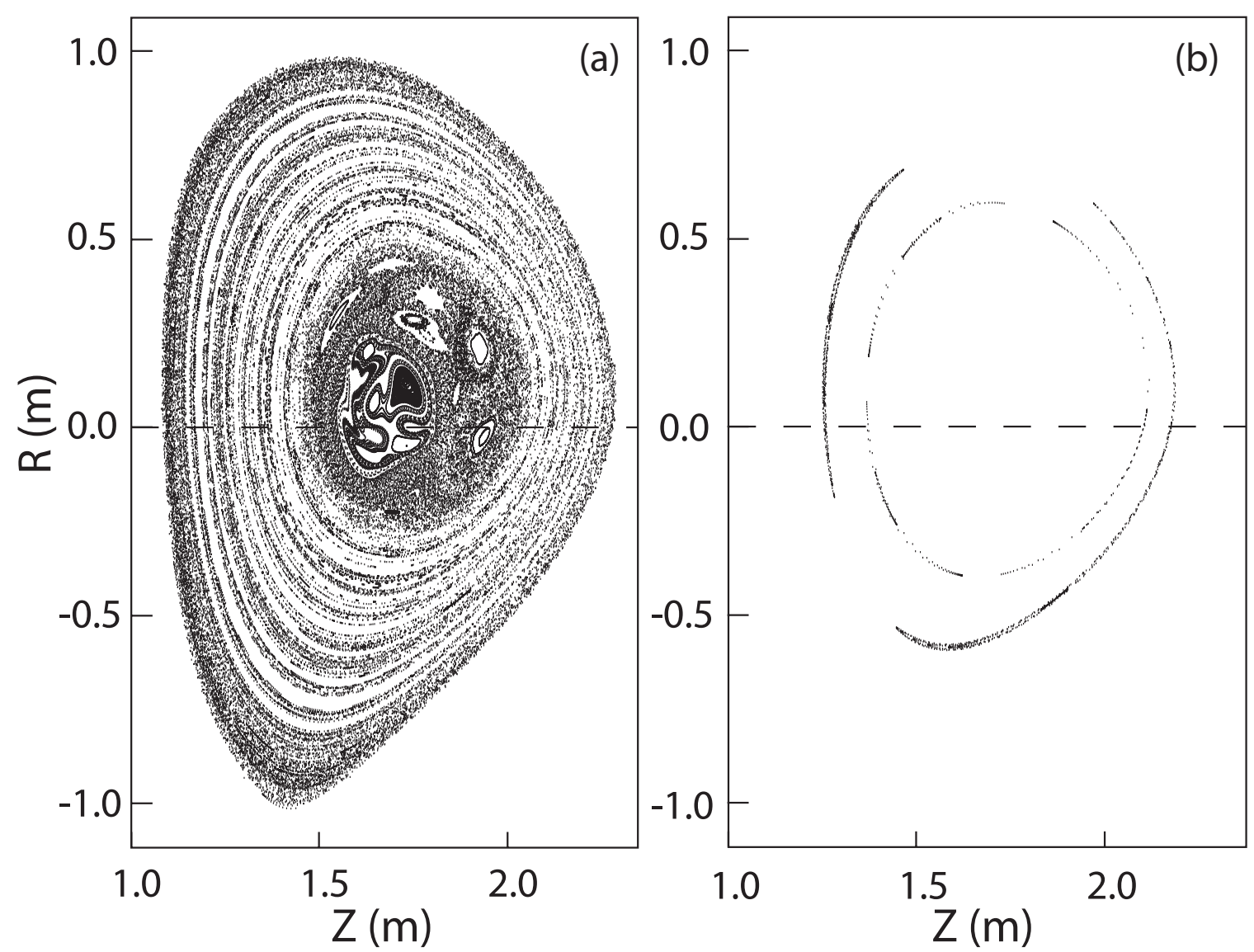

Fig. 3 Poincare surface of sections showing the $3 / 2$ and $2 / 1$ islands at the point of saturation for $\Omega \tau_{\lambda}=0.039$. The series of islands in the core are a consequence of the very low q shear after reconnection. The drven islands are very small at sawtooth saturation. 